\title{
Editorial
}

\section{Los tsunamis por Covid-19 en Perú: El primero malo, segundo peor}

\section{Covid.19 Tsunamis in Peru: First bad, second worse}

\section{$\mathrm{DOI}$}

Percy Mayta-Tristán ${ }^{1, a}$

https://doi.org/10.35434/rcmhnaaa.2021.143.1249

Perú ha sido uno de los países con mayor mortalidad durante la primera ola de COVID19 en el mundo ${ }^{(1)}$, así mismo, esta primera ola generó altas seroprevalencias que fueron medidas en Lima $^{(2)}$, Iquitos ${ }^{(3)}$, Cusco $^{(4)}$ y Lambayeque ${ }^{(5)}$ entre las más altas reportadas en la región y el mundo ${ }^{(6)}$.

Si bien aún no ha quedado claro por qué Perú ha sido tan fuertemente afectado durante la primera ola, se han planteado diversas hipótesis entre las que se incluyen la inestabilidad política con cambios de presidentes y ministros de salud que no permitió una adecuada respuesta nacional ${ }^{(7,8)}$, la alta informalidad laboral que llevó a que las personas no puedan mantener un distanciamiento social, así como las condiciones de vivienda y hacinamiento y un transporte público precario ${ }^{(8)}$, pero sobre todo un sistema de salud fragmentado, centrado en lo hospitalario, con pobre infraestructura y cobertura $^{(8-10)}$

Este sistema de salud con poca capacidad de respuesta, con uno de los más bajos números de camas en las unidades de cuidados intensivos ( $\mathrm{UCI}$ ) por habitante en la región ${ }^{(11)}$, fue uno de los factores fundamentales para una alta mortalidad. Diversos estudios en hospitales del país encontraron una mortalidad intrahospitalaria que podía llegar al 60\%, identificando como factor de riesgo para muerte una baja saturación de oxígeno al momento de la hospitalización ${ }^{(12-14)}$, debido que, por la falta de camas de hospitalización o de UCI las personas ingresaban en forma tardía.

A pesar de la alta seroprevalencia alcanzada en la primera ola, que por su magnitud fue más un tsunami ${ }^{(15)}$, se esperaba una segunda ola menos mortal; sin embargo, la segunda fue peor que la primera, estimando más de 200 mil muertos por COVID al final de ambas olas ${ }^{(16)}$. Una de las potenciales explicaciones fue la circulación predominante de las variantes lambda y gamma ${ }^{(17)}$, escasez de camas y disponibilidad de oxígeno, así como la no implementación de las lecciones aprendidas de la primera ola ${ }^{(9)}$.

Paradójicamente la mortalidad intrahospitalaria en los hospitales del Ministerio de Salud fue mayor que en la primera ola (Figura 1). Durante la segunda ola, en aquellas semanas con mayor número de ingresos hospitalarios en el Ministerio de Salud la mortalidad fue superior que en semanas con menor número de casos $(\mathrm{HRa}=4,2 ; 3.9$ $4,5)$ siendo indicativo de una rápida saturación de la cobertura hospitalaria, más aún que en este análisis no se incluyó a las personas que fallecieron sin llegar a un hospital (Mayta-Tristán, datos no publicados).

Desde julio de 2021 no se ha identificado un incremento considerable del número de casos, hospitalizaciones o muertes por COVID-19 en el territorio nacional como para denominarlo una tercera ola, esto a pesar de que la variante Delta es la predominante en el país ${ }^{(18)}$. Pero el riesgo de tener un nuevo tsunami sigue latente si llega una variante con una mayor transmisibilidad, que pueda reinfectar o infectar a vacunados y que sea tan severa como las variantes previas, pues a pesar del buen avance en la vacunación y la alta seroprevalencia que confiere inmunidad híbrida en vacunados, la pobre infraestructura del sistema de salud peruano a nivel de disponibilidad de camas,

\author{
FILIACIÓN \\ 1. Dirección General de Investigación, \\ Desarrollo e Innovación, Universidad \\ Científica del Sur. Lima, Perú. \\ a. Médico epidemiólogo. \\ ORCID: \\ 1. Percy Mayta-Tristán \\ 0000-0002-0861-6606 \\ CORRESPONDENCIA \\ Percy Mayta-Tristán \\ EMAIL \\ pmayta@cientifica.edu.pe
}

\section{CONFLICTOS DE INTERÉS}

El autor declara no tener conflictos de interés con la publicación de este artículo.

\section{COMO CITAR}

Mayta-Tristán, P. Los tsunamis por Covid19 en Perú: El primero malo, segundo peor. Revista Del Cuerpo Médico Hospital Nacional Almanzor Aguinaga Asenjo, $2021,14(3), 260-261$. https://doi.org/10.35434/rcmhnaaa.20 21.143.1249 
oxígeno y ventiladores mecánicos, sumada a la permanente inestabilidad política de estos tiempos, nos hacen susceptibles de una nueva catástrofe por COVID-19.

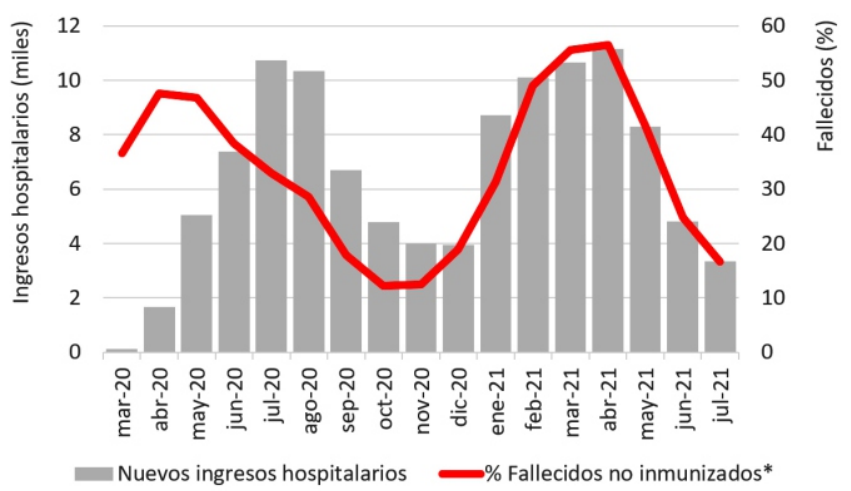

Figura 1.

Ingresos hospitalarios por COVID-19 en hospitales del Ministerio de Salud y porcentaje de fallecidos, Perú marzo 2020 a julio 2021

\section{REFERENCIAS BIBLIOGRÁFICAS}

1. Karlinsky A, Kobak D. Tracking excess mortality across countries during the COVID-19 pandemic with the World Mortality Dataset. Elife. 2021;10:e69336.

2. Reyes-Vega MF, Soto-Cabezas MG, Cárdenas F, Martel KS, Valle A, Valverde $\mathrm{J}$, et al. SARS-CoV-2 prevalence associated to low socioeconomic status and overcrowding in an LMIC megacity: A population-based seroepidemiological survey in Lima, Peru. EClinicalMedicine. 2021;34:100801.

3. Álvarez-Antonio C, Meza-Sánchez G, Calampa C, Casanova W, Carey C, Alava F, et al. Seroprevalence of anti-SARS-CoV-2 antibodies in Iquitos, Peru in July and August, 2020: a population-based study. Lancet Glob Health. 2021 Jul;9(7):e925-e931.

4. Huamaní C, Velásquez L, Montes S, Mayanga-Herrera A, BernabéOrtiz A. SARS-CoV-2 seroprevalence in a high-altitude setting in Peru: adult population-based cross-sectional study. PeerJ. 2021;9:e12149.

5. Díaz-Vélez C, Failoc-Rojas VE, Valladares-Garrido MJ, Colchado J, Carrera-Acosta L, Becerra M, et al. SARS-CoV-2 seroprevalence study in Lambayeque, Peru. June-July 2020. PeerJ. 2021;9:e11210.

6. Núñez-Zapata SF, Benites-Peralta B, Mayta-Tristan P, RodríguezMorales AJ. High seroprevalence for SARS-CoV-2 infection in South America, but still not enough for herd immunity! Int J Infect Dis. 2021
Aug;109:244-246. doi: 10.1016/j.ijid.2021.07.022. Epub 2021 Jul 11. PMID: 34260956; PMCID: PMC8272886.

7. Dargent E, Rousseau S. Perú 2020: ¿el quiebre de la continuidad? Rev Cienc Polit. 2021;41(2):377-400.

8. Villaran F, Lopez S, Ramos MC, Quintanilla P, Solari L, Ñopo H, et al. Informe sobre las causas del elevado número de muertes por la pandemia del covid-19 en el Perú. Lima: CONCYTEC; 2021.

9. Herrera-Añazco P, Uyen-Cateriano A, Mezones-Holguin E, TaypeRondan A, Mayta-Tristán P, Malaga G, et al. Some lessons that Peru did not learn before the second wave of COVID-19. Int J Health Plann Manage. 2021. DOI: $10.1002 / \mathrm{hpm} .3135$

10. Blas M, Riega P, Cuba S, Diaz C, Huamani C, Hurtado Y, et al. Atención primaria primero: integrando el primer nivel de atención, hacia un sistema nacional de servicios de salud basado en atención primaria en el Perú. Lima: INCIDE Grupo Salud; 2021.

11. Almeida F. Exploring the Impact of COVID-19 on the Sustainability of Health Critical Care Systems in South America. Int J Health Policy Manag. 2020 Jul 5. doi: 10.34172/ijhpm.2020.116.

12. Mejía F, Medina C, Cornejo E, Morello E, Vásquez S, Alave J, et al. Oxygen saturation as a predictor of mortality in hospitalized adult patients with COVID-19 in a public hospital in Lima, Peru. PLoS One. 2020;15(12):e0244171.

13. Hueda-Zavaleta M, Copaja-Corzo C, Bardales-Silva F, Flores-Palacios R, Barreto-Rocchetti L, Benites-Zapata VA. Factors associated with mortality due to COVID-19 in patients from a public hospital in Tacna, Peru. Rev Peru Med Exp Salud Publica. 2021;38(2):214-223.

14. Díaz-Vélez C, Urrunaga-Pastor D, Romero-Cerdán A, Peña-Sánchez ER, Fernández Mogollon JL, Cossio Chafloque JD, Marreros Ascoy GC, Benites-Zapata VA. Risk factors for mortality in hospitalized patients with COVID-19 from three hospitals in Peru: a retrospective cohort study. F1000Res. 2021;10:224.

15. ¡No más tsunamis! [Internet] Lima: La República; 2021. [citado: 10 de noviem bre de $\left.\begin{array}{llll}2 & 0 & 2 & 1\end{array}\right]$ D is po $n$ ible e $n$ : https://larepublica.pe/opinion/2021/09/19/no-mas-tsunamis-porpercy-mayta-tristan/

16. Perú llega a 200 mil muertes por COVID-19 desde que se inició la pandemia [Internet]. Lima: El Comercio; 2021. [citado: 10 de noviembre de $\left.\begin{array}{lllllllll}2 & 0 & 2 & 1\end{array}\right]$ D is pon ible en: https://elcomercio.pe/lima/peru-llega-a-200-mil-muertes-porcovid-19-desde-que-se-inicio-la-pandemia-del-coronavirusministerio-de-salud-nndc-noticia/

17. Vargas-Herrera N, Araujo-Castillo RV, Mestanza O, Galarza M, RojasSerrano N, Solari-Zerpa L. SARS-CoV-2 Lambda and Gamma variants competition in Peru, a country with high seroprevalence. Lancet Reg Health Am. 2022;6:100112.

18. COVID-19: INS confirma que variante delta ya es la predominante en el Perú. Lima: Andina; 2021. [citado: 10 de noviembre de 2021] Disponible en: https://andina.pe/agencia/noticia-covid19-insconfirma-variante-delta-ya-es-predominante-el-peru-862684.aspx 